\title{
Investigation of Advanced Dicing Technologies for Ultra Low-k and 3D Integration
}

\author{
A. Podpod, F. Inoue, I. De Wolf*, M. K. Rebibis, R.A. Miller, E. Beyne \\ Interuniversity Microelectronics Center (IMEC) \\ *also at Dept. Materials Engineering, KU Leuven \\ Leuven, Belgium \\ arnita.podpod@imec.be
}

The introduction and use of ultra low-k dielectric materials in an attempt to achieve better die performance brought new challenges to the wafer singulation / dicing process. Ultra low-k dielectric materials can easily get damaged during conventional blade dicing due to their brittleness. Thus, there is a need to investigate alternative or more advanced dicing techniques that can deliver successful dicing processes without inducing stress or damage. Apart from looking at the compatibility of the different dicing techniques with (ultra) low-k dielectric materials, the integration of these different dicing techniques into the different 3D-technology configurations was also investigated.

This paper reports on the post dicing results and the challenges for 3D technology for the following different dicing technologies: blade dicing, laser grooving + blade dicing, laser grooving + plasma dicing, stealth dicing, plasma dicing, and laser full cut dicing.

\section{INTRODUCTION}

The continuous demand for a better die performance has led researchers in the semiconductor field to turn towards new materials with an ultra low-k dielectric constant. These materials coupled with $\mathrm{Cu}$ interconnects pave the way for a major improvement by allowing a market-relevant reduction of the interconnect delay. However, with the different chemistries and properties of these ultra low-k dielectrics, come new challenges for wafer/chip manufacturing and packaging processes. In general, the lower the $\mathrm{k}$ value of these dielectric materials the weaker are their mechanical properties. This poses a challenge particularly on the dicing process. Ultra low-k wafers tend to peel and chip more during the dicing process than traditional low-k materials, due to their brittleness. Conventional blade dicing technique becomes unreliable and unsatisfactory at this point. Thus, the need for alternative or more advanced dicing technology/techniques. Investigation on the different dicing technologies suitable for ultra low-k wafers is a starting point into solutions for different 3D integration schemes.

The different dicing technologies investigated in this study are: blade dicing, laser grooving + plasma dicing, multi-beam laser grooving + blade dicing, plasma dicing, stealth dicing, and multi-beam laser full cut dicing. Dies processed using these different dicing technologies were subjected to various inspection and post dicing tests: die strength measurement, surface roughness measurement, surface crystallinity measurement and stress measurement.
The compatibility of the different dicing techniques to the different 3D integration schemes was looked at based from the learnings on each dicing technique throughout the course of this study. The different 3D integration schemes/ configurations considered are shown in Figure 1.

A dicing technique can be suitable to any of the different schemes shown in Fig. 1, if it can address the challenges coupled with the particular scheme: i.e. types of materials in the dicing street (i.e. presence of low-k and/or metal dummies, etc), type of the stacking/bonding method used, and bonding and other interfaces.

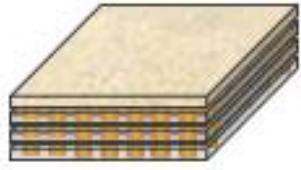

(a) Die to Die (D2D)

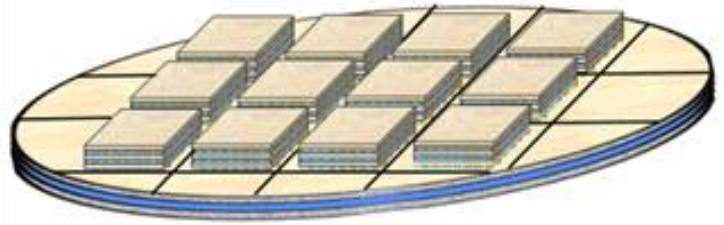

(b) Die to Wafer (D2W)

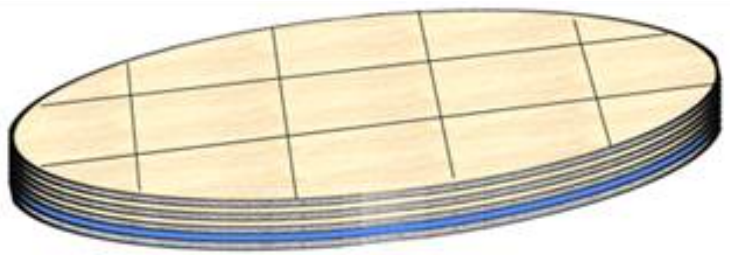

(c) Wafer to Wafer (W2W)

Figure 1. Different 3D integrations schemes / configurations: (a) die to die (D2D), die or stacked die to wafer (D2W), and wafer to wafer (W2W) 


\section{Evaluation Methodology}

\section{A. Evaluation Material}

The structures of the evaluated samples are shown in Figure 2. Note that these materials were designed taking into consideration the kind of layers present in the dicing street of the actual device wafer in one of imec's test vehicles.

Fig. 2(a) shows a schematic drawing of Test Vehicle 1 (TV1), which consists of a blanket wafer with layers of dielectrics (low-k $(\mathrm{k}=3.2)$ to ultra low-k $(\mathrm{k}=2.3)$ ). Test Vehicle 2 (TV2) shown in Fig. 2(b) contains the same dielectric layers and has in addition a $1.6 \mu \mathrm{m} \mathrm{Cu}$ metal layer in dielectric layer 4 (D4) layer. This is to consider the presence of metal dummies in the dicing street which is the case for the actual in-house device wafers.

Table I presents a summary of the evaluation material used to assess the different dicing technologies. The Si wafer thickness can be different for the different dicing techniques due to practical limitations.

\section{B. Dicing Techniques/Technologies and Process Flows}

The different dicing technologies [2] investigated in this study are: blade, plasma, stealth, multi-beam laser full cut, and combined dicing techniques namely: laser grooving + plasma, and multi-beam laser grooving + blade.

The processing steps prior and after the dicing step itself differ as dictated by certain limitations and capabilities inhouse and at the dicing demo partners. They are also dictated by what is required of the dicing process itself. As previously mentioned, the initial and final thicknesses of the evaluation materials were adapted for the different dicing techniques. All input wafers, except for plasma dicing, were thinned down (see Table I) and mounted on dicing tape with frame, prior to dicing.
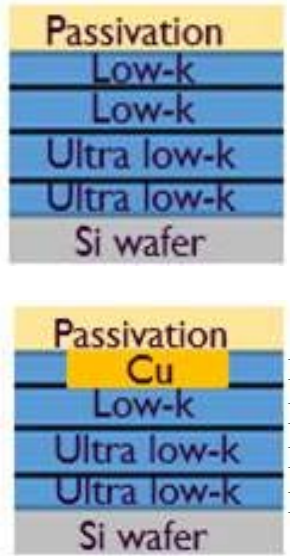

Dielectric layer 4 (D4)

Dielectric layer 3 (D3)

Dielectric layer 2 (D2)

Dielectric layer 1 (D1)

(a)

Dielectric layer 4 (D4)

Dielectric layer 3 (D3)

Dielectric layer 2 (D2)

Dielectric layer 1 (D1)

(b)

Figure 2. Structure of the test material: (a) TV1: blanket with stacked layers of dielectrics, (b) TV2: blanket with stacked layers of dielectrics $+\mathrm{Cu}$ metal
Shown in Table II is the summary of the process flow for each dicing technology investigated in this study. It can be seen that only in the Plasma dicing technique, the flow started with a full thickness wafer. In fact a dicing before grinding (DBG) method is used for this case. The main reason for utilizing the DBG method is that the resist coating/developing and the removal of resist after partial dicing in a ring frame configuration cannot be done in-house.

Keeping these different process flows in mind for the different dicing technologies is useful in considering suitable dicing techniques for different 3D integration schemes.

At the end, the wafers were singulated into dies with $10 \times 10 \mathrm{~mm}^{2}$ size, with different thicknesses as shown in Table I.

TABLE I. SUMMARY OF EVALUATION MATERIAL DESCRIPTION PER DICING TECHNOLOGY

\begin{tabular}{|l|c|c|}
\hline \multicolumn{1}{|c|}{ Dicing Technology } & $\begin{array}{c}\text { Final Si-die } \\
\text { Thickness }(\boldsymbol{\mu m})\end{array}$ & $\begin{array}{c}\text { Wafer } \\
\text { Material } \\
\text { Type }\end{array}$ \\
\hline Blade (in-house) & $100 / 50$ & TV1 / TV2 \\
\hline Plasma & 75 & TV1 \\
\hline Stealth & 50 & TV1 \& TV2 \\
\hline Stealth & 100 & TV1 \& TV2 \\
\hline Laser Grooving + Plasma & $100 / 50$ & TV1 / TV2 \\
\hline Multi-beam Laser Grooving + Blade & 100 & TV1 \& TV2 \\
\hline Multi-beam Laser Full Cut & 100 & TV1 \& TV2 \\
\hline Multi-beam Laser Full Cut & 50 & TV1 \& TV2 \\
\hline
\end{tabular}

TABLE II. Process Flow SUMMARY For The DifFERENT Dicing TECHNOLOGIES/TECHNIQUES

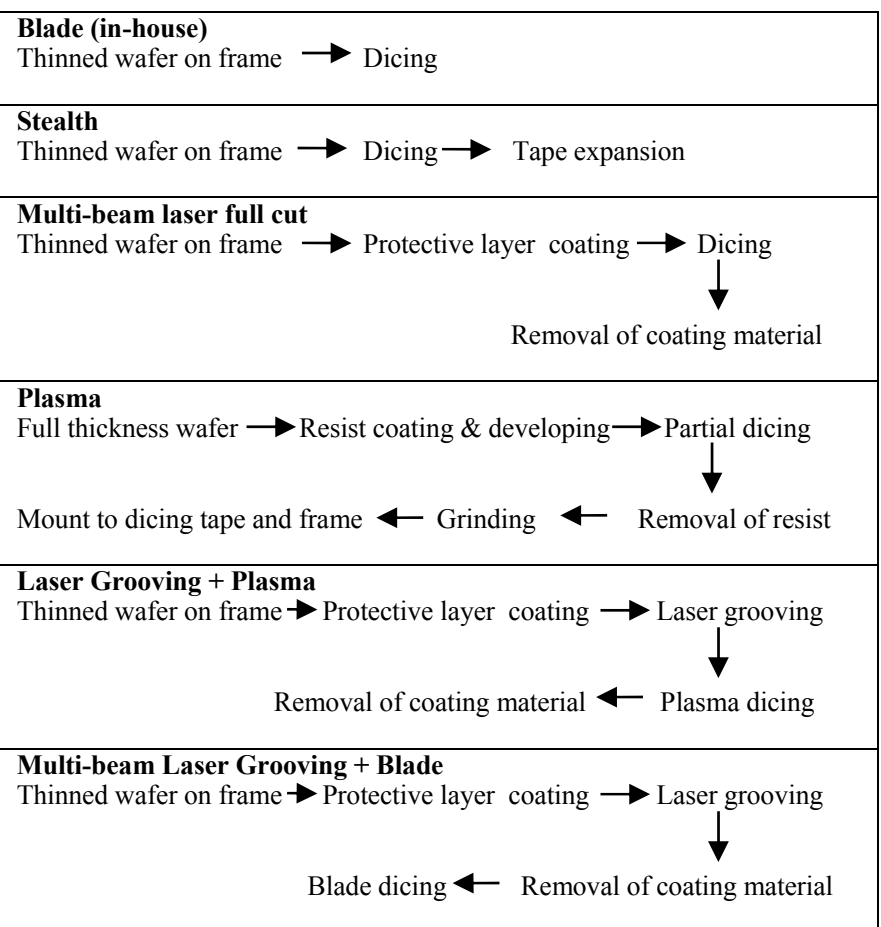




\section{Inspection and Post Dicing Tests}

All dies processed using the different dicing technologies were subjected to visual inspection using a table top high magnification microscope. Thirty (30) pieces of dies from different locations within the wafer (north, south, west, east, and center) were inspected on the front and back side for cracks, chippings and any sign of damage. Side wall inspection was done on selected dies with worst chipping/crack based from the front and back side inspection. Next, Scanning Electron Microscopy (SEM) and Energy Dispersive X-ray (EDX) analysis were done to see in detail the crack/s, peel-off and possible crack propagation.

One TV1 die from each different dicing technology was analyzed using Transmission Electron Microscope (TEM) to study in more detail structural damage, if present. The side wall surface roughness was measured on approximately a $50 \times 30 \mu \mathrm{m}^{2}$ scan area below the deposited dielectric layers, using an optical profilometer on. Furthermore, the stress on the die edge and the stress decay from the die edge moving towards the die center were measured using a Scanning Infrared Stress Explorer (SIREX). This technique is based on stress-induced changes in the polarization of IR-light [3]. It measures the difference in the in-plane normal stresses $(\Delta \sigma)$. The scan area for stress at the edge is $300 \times 300 \mu \mathrm{m}^{2}$, while the stress decay was done within a strip area of $300 \times 5 \mu \mathrm{m}^{2}$ with stacks of ten (10) strips. The SIREX measurement was done from the die backside. In addition, micro-Raman spectroscopy analysis was done on the side wall of the TV1 dies processed using the different dicing technologies. Micro-Raman spectroscopy results give indication on the stress and damage induced by the dicing technique.

All inspected dies were tested for die strength using the Plate on Elastic Foundation Test (PEFT) method [6]. Both TV1 and TV2 samples were tested with the dielectrics facing up. The PEFT method is not a JEDEC standard for die strength. However, it delivers a consistent result, based from the Displacement vs Load plots behavior, by eliminating handling and alignment issues particularly in dealing with small and thin dies. Shown in Figure 3 is the in-house PEFT method set-up. This shows its difference from the $4 \mathrm{pt}$ bending test method set-up available in-house. The silicone foundation ( $\sim 10 \mathrm{~mm}$ thick) replaces the $4 \mathrm{pt}$ bending two-pin stage, and eliminates the critical manual loading and alignment of the small thinned die. . Before deciding on using PEFT method for this study, some initial tests were done: 1) the in-house 4pt and PEFT set-up results (Displacement vs Load curves) were compared. This was done to assess the consistency of the PEFT method. 2) The die strength value (load at fracture (in $\mathrm{MPa}$ ) obtained with PEFT was compared with the value obtained using $3 \mathrm{pt}$ bending test done at one of the dicing demo partners. This was done for dies prepared using blade and laser grooving + plasma dicing technique.

The Displacement vs Load curves obtained using the PEFT method give the deformation and force at which the die break. This does not give outright the die strength value of the dies.

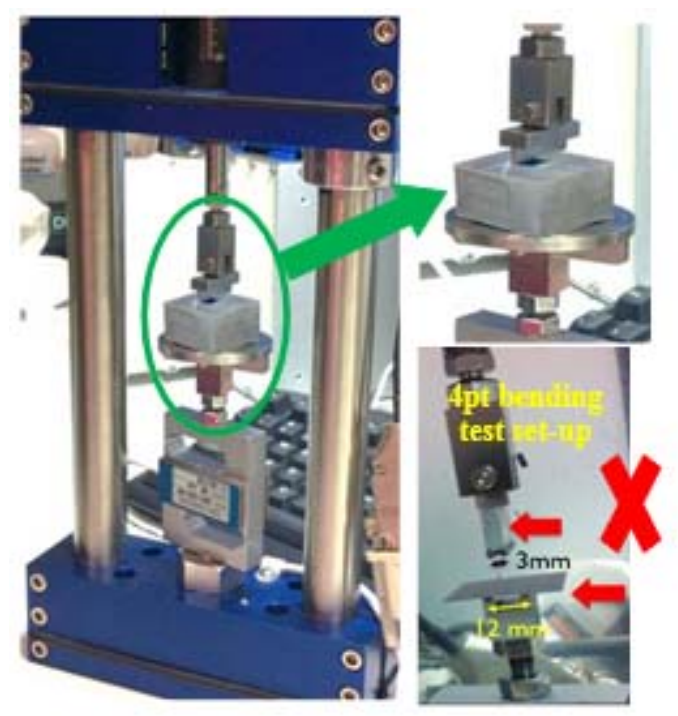

Figure 3. PEFT method in-house set-up. This highlights the difference in sample holder/platform from 4 pt bending test set-up available in house, which eliminates the difficultly in loading and alignment.

In order to calculate the die strength values, a PEFT finite element simulation was done for different die thicknesses $(50 \mu \mathrm{m}, 75 \mu \mathrm{m}$ and $100 \mu \mathrm{m})$ providing the theoretical relation between applied deformation and induced stress. The different relations between applied deformation $\left(\mathrm{U}_{\mathrm{y}}\right.$ (in $\left.\mu \mathrm{m}\right)$ where $y$ is the vertical direction in Fig. 3) and induced inplane stress $\left(\sigma_{\mathrm{xx}}\right.$, in $\left.\mathrm{MPa}\right)$ for $50 \mu \mathrm{m}, 75 \mu \mathrm{m}$ and $100 \mu \mathrm{m}$ thick dies are presented in Eq. (1-3), respectively. $\sigma_{\chi \chi}$, calculated from $U_{y}$ at the breaking moment of the die during PEFT is considered as the die strength.

$$
\begin{aligned}
& \sigma_{\chi \chi}=2.66 U_{y} \\
& \sigma_{\chi \chi}=2.852 U_{y} \\
& \sigma_{\chi \chi}=1.846 U_{y}
\end{aligned}
$$

The effect of the different dicing techniques on another important process, the die pick-up process, was also checked. This is required to ensure that dicing does not induced die pick-up issues. For example in the case of laser grooving + plasma, wherein plasma dicing was done on a wafer that was mounted on dicing tape, the die pick-up test was done to see whether the plasma does not damage or react with the dicing tape, resulting to failure in die pick-up process. Another example is for the case of stealth dicing, where the die pickup test was performed to assess whether the dicing tape is not expanding too much preventing good die pick-up. 


\section{RESULTS AND DISCUSSION}

\section{A. Blade Dicing (In-House)}

Shown in Figure 3 are representative optical microscope images of the front and back side of the dies for both TV1 and TV2 materials. Dielectric peeling and chipping can be observed on both evaluation materials. These are typical issues of the blade dicing technology. It important to note that chipping dimensions were not measured on every die in this study.

EDX and side wall SEM analysis are shown in Figure 4 for TV2 dielectric peeling. The results suggest that peeling occurred in the D4 layer or in between the D4 and D3 layer.

The side wall SEM of one of the dies from TV1, shown in Figure 5, shows a crack in the low-k D2 layer.
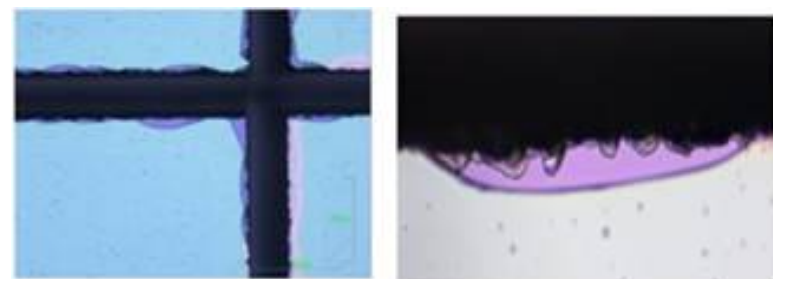

(a) TVI optical images on front side
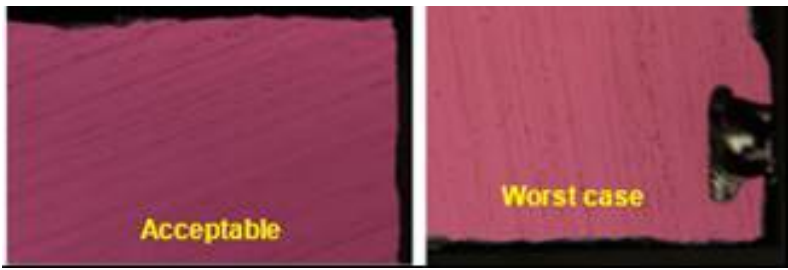

(b) TV1 optical images on back side
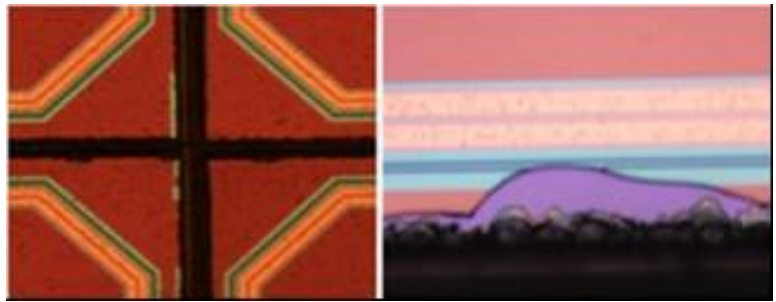

(c) TV2 optical images on front side

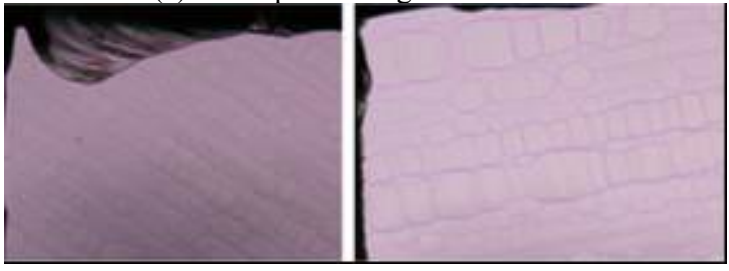

(d) TV2 optical images on back side

Figure 3. Optical images for both TV1 and TV2 materials showing the front and back side of the dies. The images shows dielectric peeling and chipping on both materials.
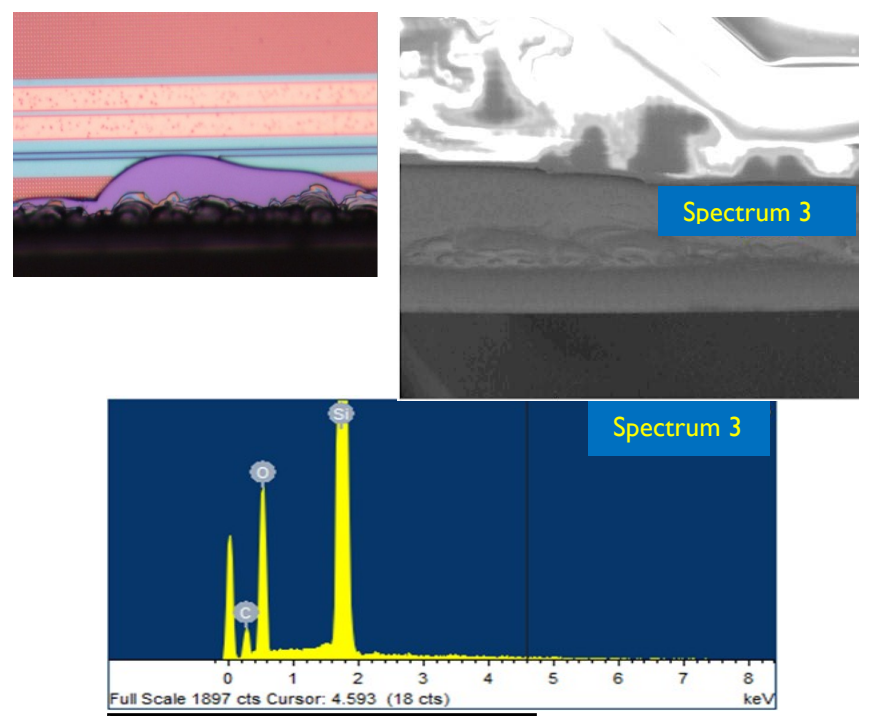

\begin{tabular}{|lll|}
\hline Element & Weight & \multicolumn{1}{c|}{ Atomic } \\
& $\%$ & $\%$ \\
C K & 16.66 & 23.82 \\
O K & 31.80 & 34.174 \\
Si K & 68.75 & 42.04 \\
\hline
\end{tabular}

(a) Top EDX analysis on dielectric peel. Shows exposed SiO layer.
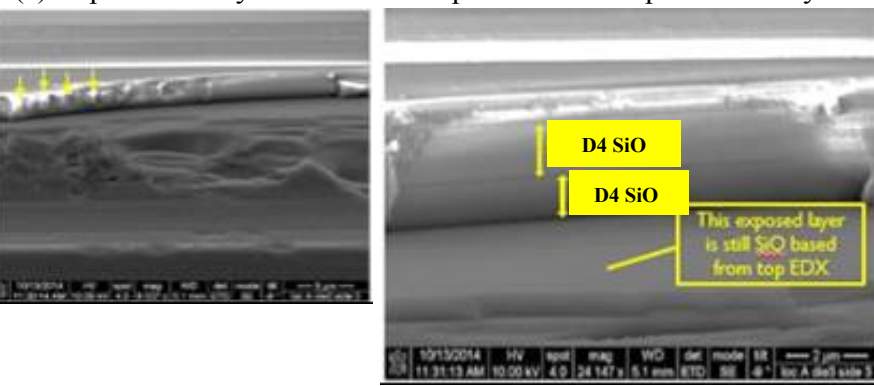

(b) Side wall SEM analysis of dielectric peel

Figure 4. Top EDX and side wall SEM analysis on TV2 dielectric peel. EDX scan show an exposed $\mathrm{SiO}$ layer. Side wall SEM suggests that peel occurred in D4 layer or in between D4 and D3 layer.

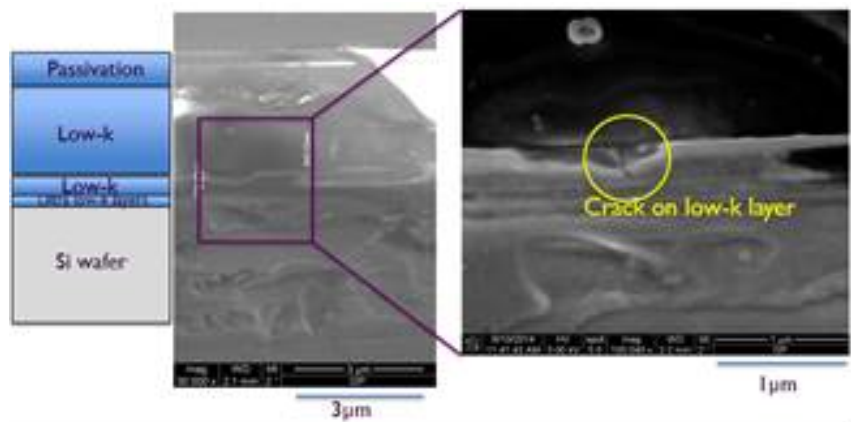

Figure 5. Side wall SEM in TV1 material. A crack on low-k D2 layer was observed.

It is clear from these results that blade dicing has a high probability to induce damage in the low-k layer.

Optical profilometer results of the surface roughness gave an average roughness within an area of $\sim 50 \times 30 \mu \mathrm{m}^{2}$ of $594 \mathrm{~nm}$. 

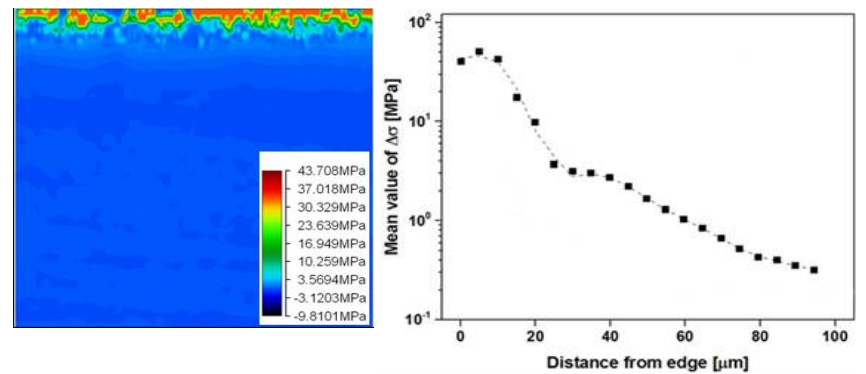

(a) SIREX results: in-plane normal stress difference $(\Delta \sigma)$ on the edge (left) and $\Delta \sigma$ decay (right)

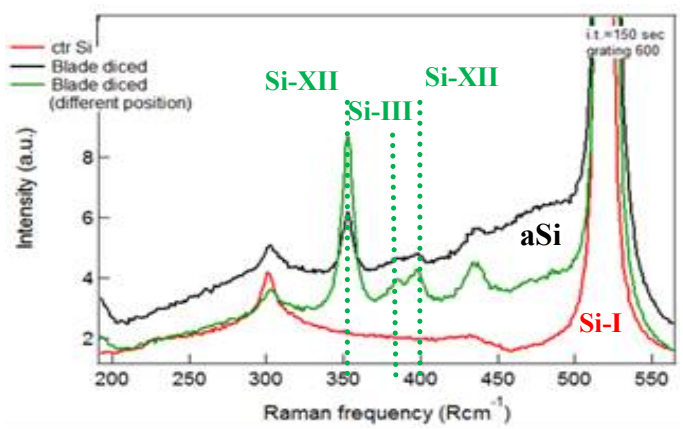

(b) Micro-Raman curves showing different Si phases on die singulated by blade dicing compared with control $\mathrm{Si}$ (ctr $\mathrm{Si})$

Figure 6. Stress related measurements from (a) SIREX, (b) and micro-Raman spectroscopy.

Stress related measurements from SIREX and Raman spectroscopy are presented in Figure 6.

In Fig. 6(a) left side, SIREX shows an approximate $37 \mathrm{MPa}$ difference in normal stresses on the die edge. The right side of Fig. 6(a) shows the mean value of $\Delta \sigma$ from the edge scanning towards the center of the die. The mean stress value decreases moving towards the center, which is an expected result, and stress starts to really go down at approximately $80 \mu \mathrm{m}$ from the edge.

Micro-Raman spectroscopy curves in Fig. 6(b), show the appearance of different $\mathrm{Si}$ phases (Si-III and $\mathrm{Si}-\mathrm{XII}$ ) on the blade diced sample [1]. These peaks can occur when high mechanical stress or pressure has been applied onto the Si. In addition, also the presence of an amorphous $\mathrm{Si}(\mathrm{aSi})$ peak indicates that there is a change in the crystal structure of $\mathrm{Si}$.

The mean value of the die strength obtained from PEFT is $115 \pm 5 \mathrm{MPa}$. The manner of die breakage after test was also investigated as presented in Figure 7 . For these samples, the majority of the dies broke in the middle with a splinter as shown in Fig. 7(a).

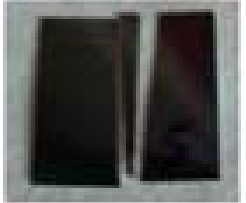

(a)

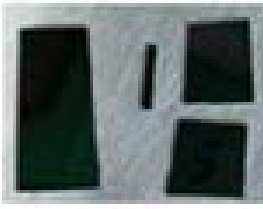

(b)

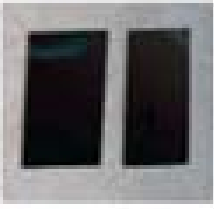

(c)

\section{B. Plasma Dicing}

Shown in Figure 7, are representative optical images (front, back and side wall view) of dies processed using plasma dicing. Dielectric peeling was observed on the front side of the dies. However, this happened during the manual tape transfer from grinding tape to dicing tape. The dies looked flawless on top and bottom after the plasma etch. Notice the presence of the Si dusts on the side wall surface shown in Figure 7(c). This was introduced during grinding and it was not possible to completely clean this because of the very small gap/width in between the dies $(10 \mu \mathrm{m}$ width/gap).

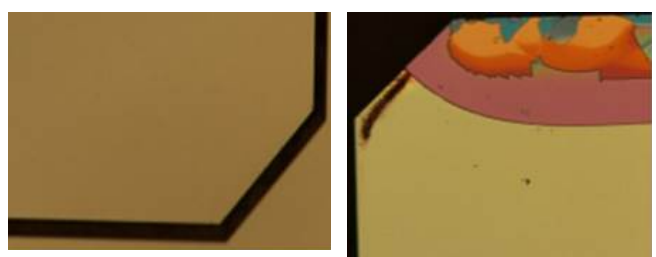

(a) Optical images front side view.

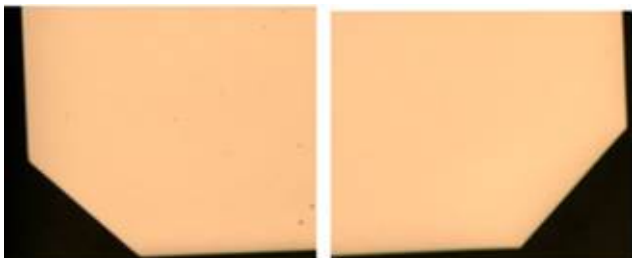

(b) Optical images back side view

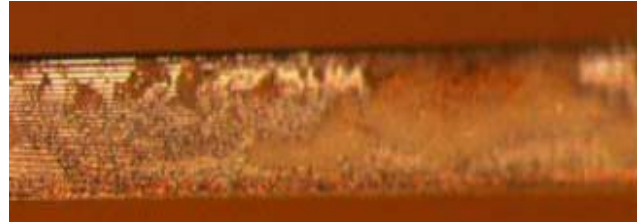

(c) Optical side wall surface image

Figure 7. Representative optical images of plasma diced samples at front, back and side wall view.

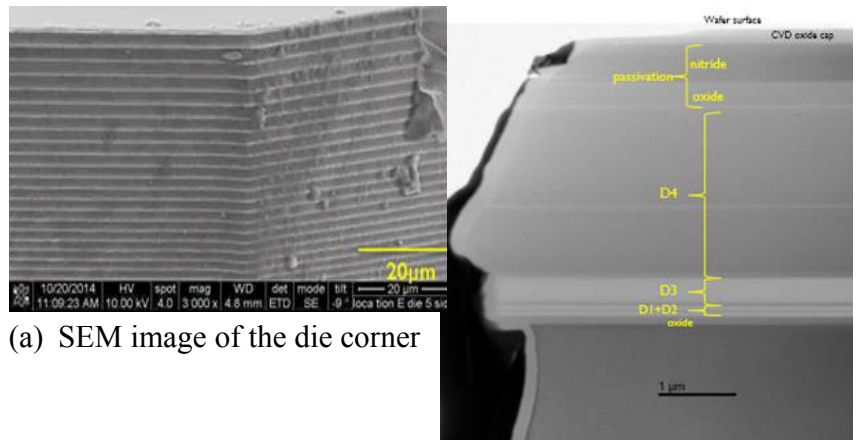

(b) TEM image

Figure 9. SEM and TEM image of TV1 sample. No crack observed.

Figure 7. Die fracture after PEFT test on blade diced dies. 
SEM and TEM images shown in Fig. 9(a) and (b) respectively, show no crack in dielectric layers. Although, sample preparation for TEM was a challenge because of the rough edges (scallops). Optical profilometer measurements give an average roughness of approximately $207 \mathrm{~nm}$ in an area $50 \times 36 \mu \mathrm{m}^{2}$.

Stress related measurements, comparing plasma dicing with blade dicing, shown in Fig. 10 show a lower stress induced by plasma dicing on the dies. Stress $\Delta \sigma$ at the edge has an approximate value of $2.8 \mathrm{MPa}$. The $\Delta \sigma$ decay drops faster than that of blade dicing as shown in Fig. 10(a). The increase of the mean $\Delta \sigma$ value at around $30 \mu \mathrm{m}$ towards the center was produced due to difficultly of measurement as the surface has particles on it. The trend is expected to maintain a low value. The micro-Raman spectrum in Fig. 10(b) shows a much higher intensity of the plasma diced sample compared with the blade diced one. A decrease in intensity and broadening peak indicates damage. Also the Raman frequency shift $\left(\mathrm{cm}^{-1}\right)$ from the one of a stress-free control crystalline $\mathrm{Si}$ sample (ctr $\mathrm{Si}$ ) is more negative for the blade dicing and nearly zero for the plasma dicing. This indicates induced stress or damage in the blade diced sample, and good quality $\mathrm{Si}$ in the plasma diced sample.

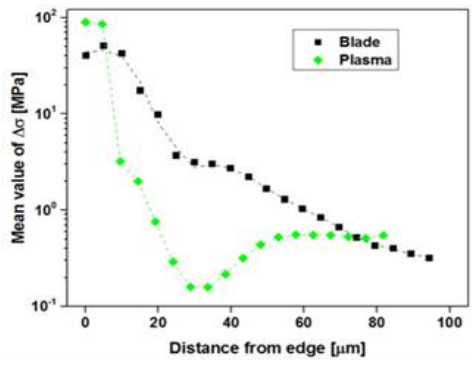

(a)

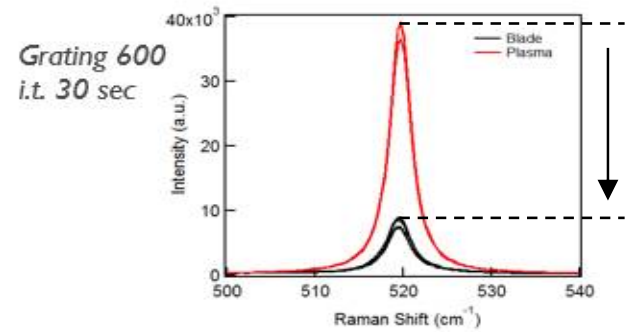

(b)

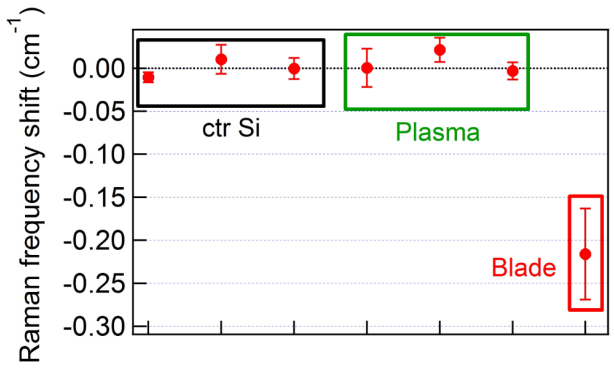

Figure 10. Stress measurements comparison of plasma vs blade dicing: (a) Stress from the edge towards the center (stress decay) obtained from SIREX, (b) Micro-Raman spectroscopy results, and (c) Si Raman frequency $\left(\mathrm{cm}^{-1}\right)$.

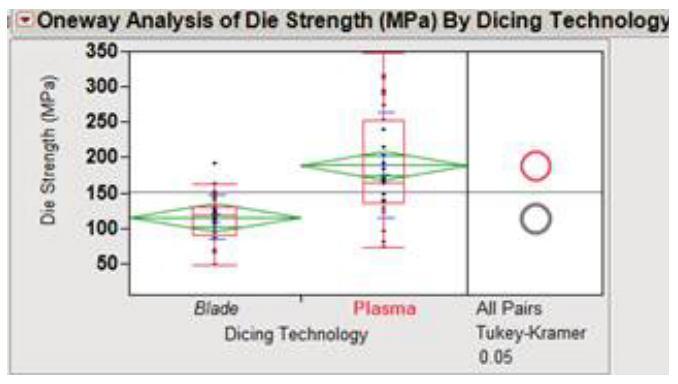

(a) Die strength (MPa) plot for Blade and Plasma Dicing

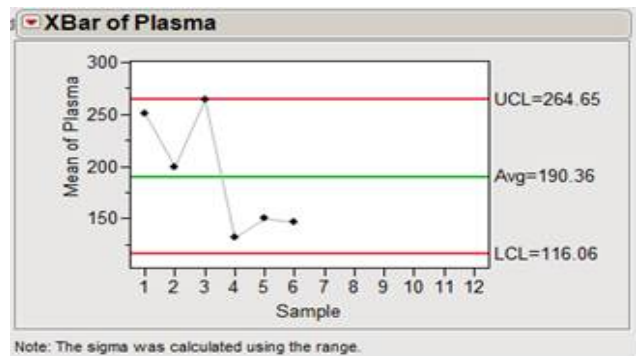

(b) XBar control chart plot for Plasma diced samples

Figure 11. Calculated die strength values for Blade and Plasma dicing techniques. Higher die strength observed for plasma dicing technique.

The die strength results for blade and plasma dicing are compared in Fig. 11 plotted. It can be seen that there is a significant increase (from the Turkey-Kramer JMP test) in the mean value for plasma diced samples. This is logical since lower stress and damage was induced using the plasma dicing technique compared with blade dicing. Stress measurement and die strength results are in synced. Although a larger spread in data can be observed on plasma diced sample, the data are not erratic as shown in JMP XBar control chart in Fig. 11 (b).

\section{Stealth Dicing}

Representative optical microscopy images in front, back and side wall view are shown in Figure 12. In Fig. 12a and $\mathrm{b}$, peeling is shown to occur on the front side and there was no chipping observed at the backside. The wider gap in between dies is evident on the left side image of Fig. 12(a). This is brought by the expansion step during the stealth dicing process. Too much expansion will cause the dicing tape to sag and this might lead to problems during die pickup. There was however no die pick-up issue encountered in this study after the stealth dicing process. The left and right side images of Fig. 12(c), show the side wall view of a die with a thickness of $50 \mu \mathrm{m}$ and $100 \mu \mathrm{m}$, respectively. The stealth dicing layer is clearly visible. It can be seen that there are wider area (top and bottom) without the laser path for the thicker die. This contributes to a smoother side wall surface as obtained from optical profilometer scan. The average roughness was $408 \mathrm{~nm}$ and $50 \mathrm{~nm}$ for a die thickness of $50 \mu \mathrm{m}$ and $100 \mu \mathrm{m}$, respectively. 


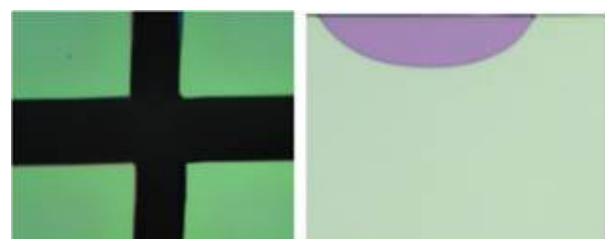

(a) Microscope images on the die front side. Peeling observed

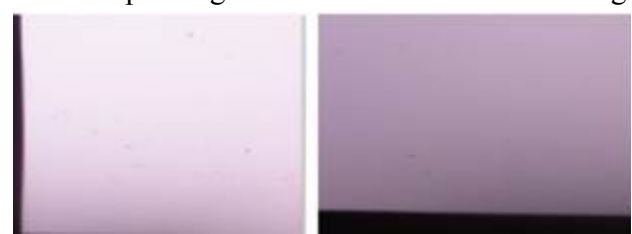

(b) Microscope images on the die back side. No chipping observed
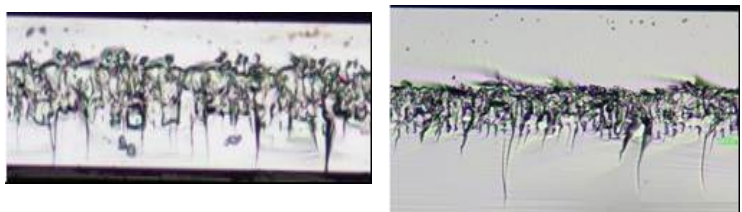

(c) Microscope images on the die side wall: $50 \mu \mathrm{m}$ thickness (left), $100 \mu \mathrm{m}$ thickness (right). The laser path of the Stealth dicing is clearly visible

Figure 12. Representative microscope images of the die processed by stealth dicing: (a) front side, (b) back side, and (c) side wall view.

No crack was found in the low-k or ultra low-k layers on dies singulated by stealth dicing. Some typical SEM and TEM images presented in Fig. 13 show good, crack free layers. There is some damage in the boxed area of the TEM image. However, no crack or propagating crack is seen. This damage is most likely related to the rough areas seen after the expansion step in the stealth dicing process.

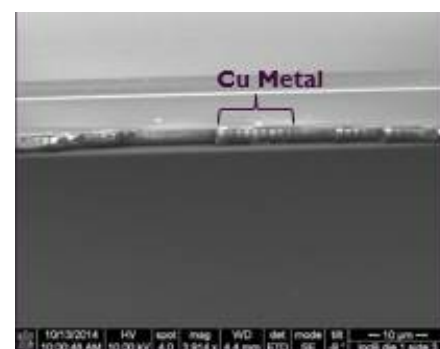

(a) SEM side wall image

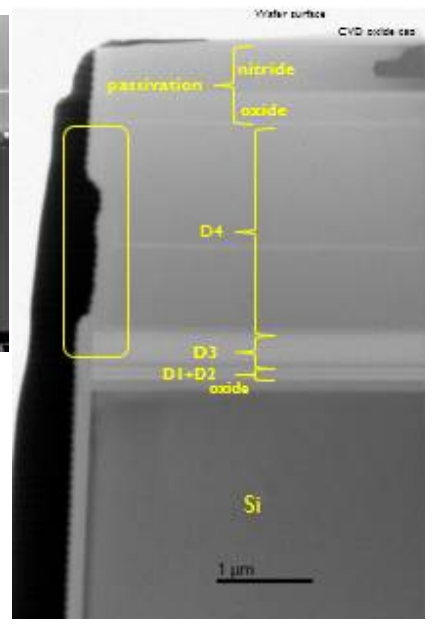

(b) TEM image
Figure 13. SEM and TEM images. No cracks found on layers.
The SIREX $\Delta \sigma$ value at the die edge was approximately $5 \mathrm{MPa}$ and the stress decay of the stealth diced sample also drops faster than in the blade diced sample, as shown in Fig. 14(a). Fig. 14(b) shows the Raman intensity for thicknesses $50 \mu \mathrm{m}$ and $100 \mu \mathrm{m}$ along with the blade technology. Note that there are two Raman points for dies singulated by stealth dicing: points within the $\mathrm{Si}(\mathrm{SD} 50 \mathrm{Si}, \mathrm{SD} 100 \mathrm{Si})$ area and points within the laser path area (SD50 Laser, SD100 Laser). Regardless of die thickness, Raman intensity for stealth dicing is higher than blade indicating a better quality of the Si crystal. In addition, the Raman frequency $\left(\mathrm{cm}^{-1}\right)$ is shown in Fig. 14c. In an area within the laser path the Raman peak of Si shifted to the positive side for $50 \mu \mathrm{m}$ thickness. This suggests a compressive stress induced by stealth dicing. On the other hand, Raman frequency $\left(\mathrm{cm}^{-1}\right)$ within the laser area shifted very slightly on negative side for $100 \mu \mathrm{m}$ thickness indicating a slight damage locally.

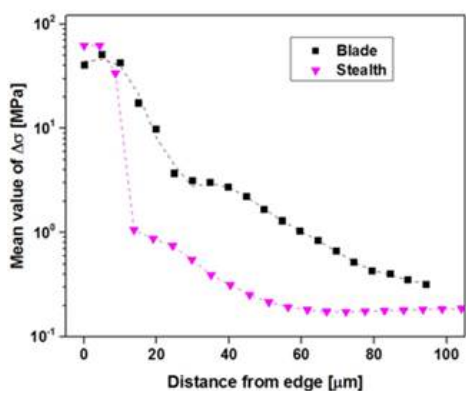

(a)

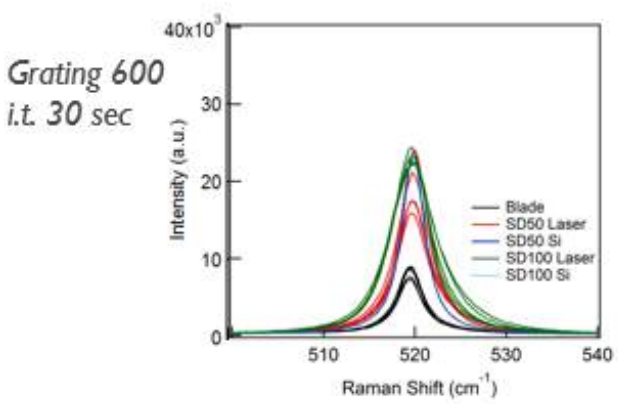

(b)

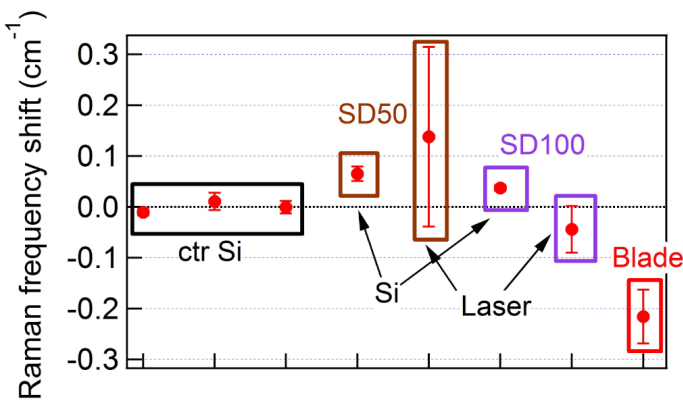

(c)

Figure 14. Stress measurements: (a) Stress from the edge towards the center (stress decay), (b) Micro-Raman spectroscopy scan, and (c) Raman frequency $\left(\mathrm{cm}^{-1}\right)$ scan. Lower stressed induced by stealth dicing compared to blade. 
Contrary to what is expected from these results, the die strength values show no significance difference between blade and stealth dicing technology. This can be due to different factors affecting the die strength, in this case most likely the grinding process. Indeed, wafers for blade dicing were thinned in-house while the wafers for stealth dicing were thinned at the dicing partner's site.

\section{Multi Beam Laser Full Cut}

There were no peeling, large chipping or laser related damages observed on the front side of dies singulated using multi-beam laser full cut. However, die chippings were seen at the back of the dies. Representative photos are shown in Figure 15 taken at 30x objective magnification. The chipping can be minimized by optimization of the dicing process.

Looking at the side wall of the dies, it is not very distinguishable if there are small cracks or damage due to the rough path of the laser. No unusual appearance could be observed from the SEM image shown in Figure 16. TEM analysis was used to study this surface in more detail, a result is shown in Figure 17.

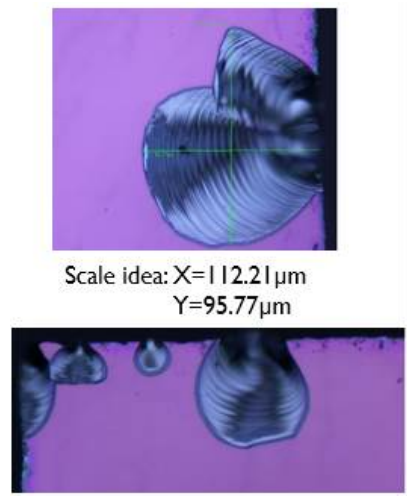

Figure 15. Back side chipping seen after multi-beam laser full cut dicing.

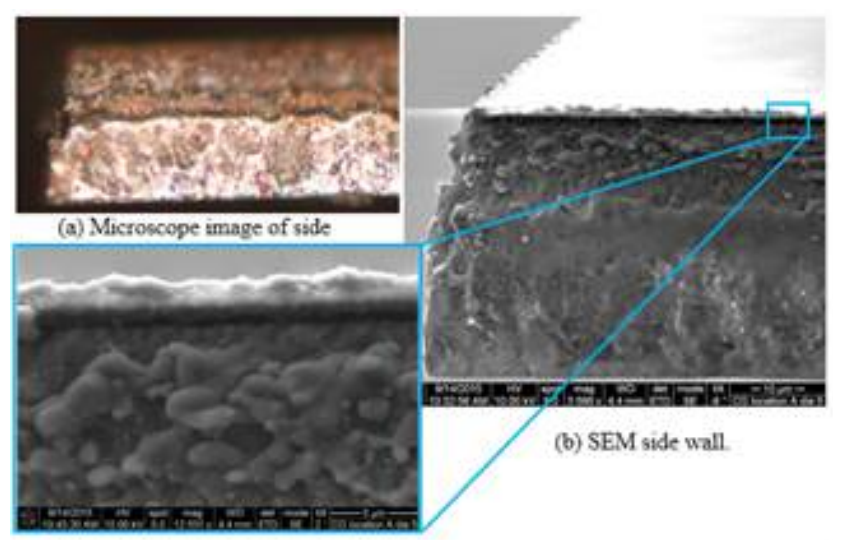

Figure 16. Side wall images: (a) microscope image and (b): SEM images.
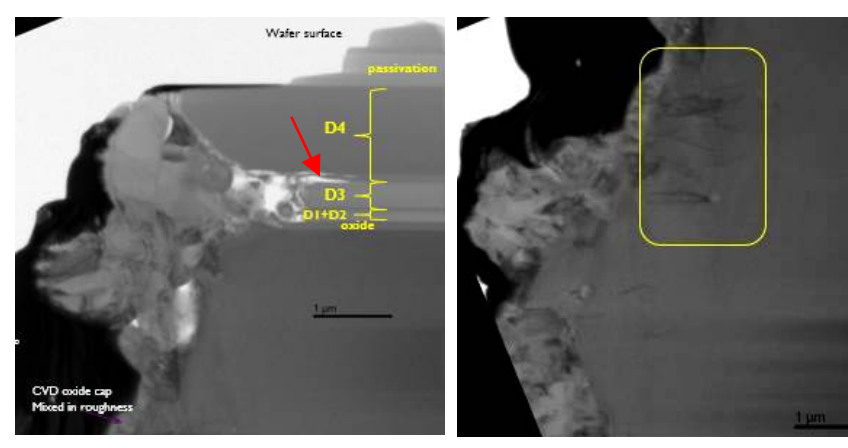

Figure 17. TEM image of die singulated using multi-beam laser full cut.

Pointed by an arrow in the left photo of Fig. 17 is a void seen between the D4 and D3 layer. This void can indicate that there is damage in the layers, induced by the dicing. In addition, dislocations are observed in $\mathrm{Si}$ as indicated by the boxed area of the right photo in Fig. 17.

Stress measurement on the edge done using SIREX gave a value of $\Delta \sigma=26 \mathrm{MPa}$, which is still lower than the $37 \mathrm{MPa}$ obtained from blade dicing. From Fig. 18(a), it can be observed that the stress decay does differ much from the blade dicing case. However, there is a noticeable decrease in the mean $\Delta \sigma$ value at approximately $20 \mu \mathrm{m}$ from the die edge.

There were two points taken for micro-Raman spectroscopy measurements on the side wall of a $50 \mu \mathrm{m}$ thick die: one is near the top part of the side wall of the die and the other one is near the bottom. The Raman intensity plot in Fig. 18(b) shows the appearance of amorphous Si for the laser full cut sample. This is logical as there will be melted Si during the laser process. An indication of damage induced on the die can be seen in Fig. 18(c), wherein there is a negative shift with respect to control $\mathrm{Si}$ (ctr $\mathrm{Si}$ ) in the die diced using laser full cut. The same case as that of the blade dicing.

The measured mean die strength for dies singulated by laser full cut is $99 \mathrm{MPa}$, which is significantly lower than the blade results, based on the Turkey-Kramer JMP software.

\section{E. Multi-Beam Laser Full Cut + Blade}

For the front side of the die, the same observations were made for the combination of multi-beam laser cut + blade dicing as the ones for multi-beam laser full cut: no damage induced and no peeling. However, the backside, as expected, has a lot of chippings. This is similar to what was observed for blade dicing, which is of course logical since this is a combination of blade dicing and laser grooving.

Figure 19(a) shows the SEM side wall pictures and corresponding Raman spectra for the laser grooved zone and the blade diced zone. The different phases of $\mathrm{Si}$ are again observed in the blade diced zone, but are not present in the laser grooved zone. These peaks indicate a temporary 
occurrence of very high local stresses during blade dicing, resulting in phase changes of the Si crystal.

The stress $\Delta \sigma$ at the die edge has a value of $26 \mathrm{MPa}$. On the other hand, the $\Delta \sigma$ decay is relatively faster than that of blade dicing, as shown in Fig. 19(b). However, this does not translate directly to the measured die strength value. There is no significant difference between the die strength of the die singulated using blade dicing and the one from the combined dicing technique. This basically can be due to the fact that the blade dicing part covered the largest dicing depth in this die singulation process. The laser grooving part was just $16 \mu \mathrm{m}$ deep of the die thickness.

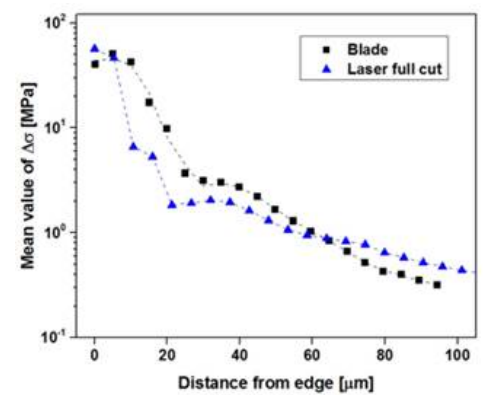

(a) Stress decay measurement for blade diced and multi-beam laser full cut diced sample.

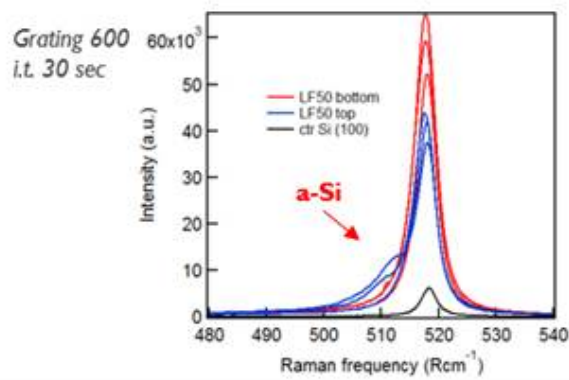

(b) Micro-Raman intensity measurement for blade diced and multi-beam laser full cut diced sample.

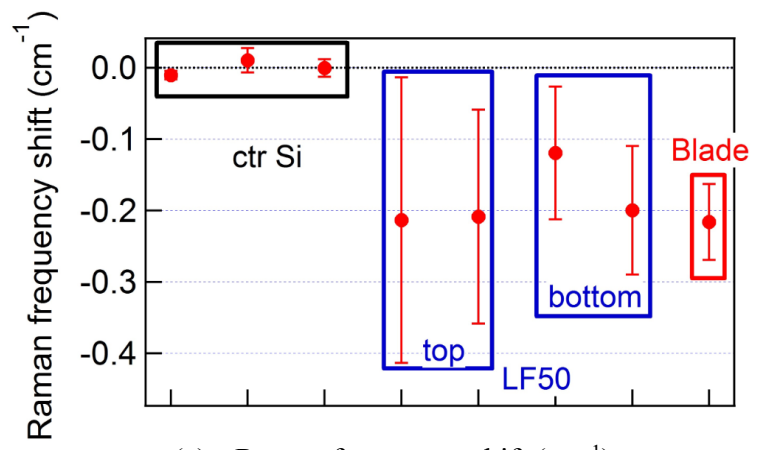

(c) Raman frequency shift $\left(\mathrm{cm}^{-1}\right)$

Figure 18. Stress measurements: (a) Stress from the edge towards the center (stress decay), (b) Micro-Raman scan, and (c) Raman frequency $\left(\mathrm{cm}^{-1}\right)$ scan. Damage induced on die by multi-beam laser full cut technique.

\section{F. Laser Grooving + Plasma Dicing}

As discussed in part IIA, in this combined laser grooving and plasma dicing technique, the plasma etch was done on a thinned wafer mounted on dicing tape and metal frame. In this particular case, the die pick-up test was done on a blanket $50 \mu \mathrm{m}$ thinned wafer before proceeding with the dicing evaluation on the test materials. This was performed to assess the compatibility of the plasma process with the standard dicing tape being used in-house. No problem was encountered during the die pick-up process and there was no indication that the dicing tape was damaged by the plasma etch step.

As shown in Fig. 20(a), laser induced damage was observed on the front side. Fig. 20(b) shows representative photos from the die backside showing minimal chipping. The chipping can be due to over etching during the plasma process or can also be due to handling, specifically if the chippings are at the corners of the die. Side wall images from optical microscope and SEM are in Fig. 20(c). There was no distinguishable damage or crack observed.

TEM analysis is shown in Fig. 21. Pointed by an arrow is a void extending as a crack in between D3 and D4, observed in the laser grooved zone. This damage along with laser damage on top and chipping at the back are indications that the laser grooving and plasma etch process need to be optimized.

An approximately $\Delta \sigma=30 \mathrm{MPa}$ stress is measured using SIREX on the die edge. The stress drastically drops at $\sim 20 \mu \mathrm{m}$ distance from the die edge as can be seen in Fig. 22(a). A better Si quality compared to blade dicing is suggested by the higher Raman peak intensity shown in Fig. 22(b). In this case, there are again two points for microRaman spectroscopy scans: one point at the laser grooved area and another one at the plasma etched area. Raman frequency shift $\left(\mathrm{cm}^{-1}\right)$ results in Fig. 22(c) show a very small positive drift that indicates a small compressive stress.

The mean value of the die strength does not show a significant difference from blade dicing. However, the distribution of the data is higher for this combined dicing technique compared with blade dicing, indicating a good agreement with the stress measurements. 


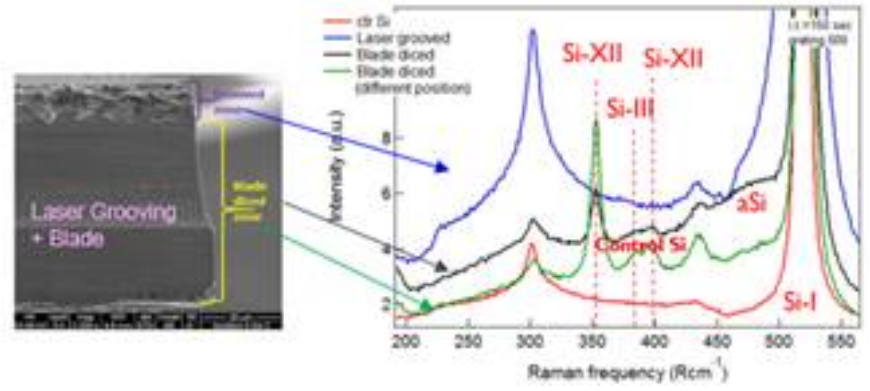

(a) SEM side wall image with micro-Raman scan

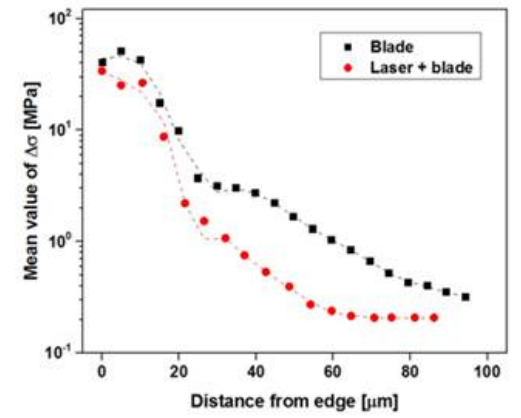

(b) Stress decay measurement blade and combined technique

Figure 19. Stress measurements: (a) side wall SEM with microRaman scan on the laser grooved and blade diced zone, and (b) Stress decay measurement of sample singulated by blade and combined technique.
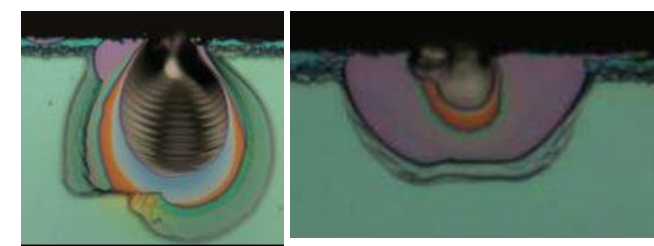

(a) Damage induced by laser on the die front side

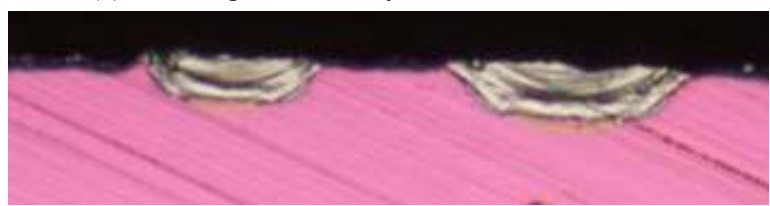

(b) A view from die back side. Minimal chipping
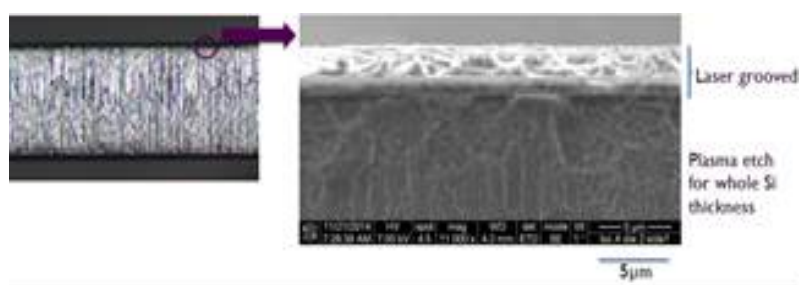

(c) Side wall optical microscope image (left) and SEM image (right). No crack observed.

Figure 20. Representative images of the die dieced using combined laser grooving + plasma dicing technique: (a) front side view showing laser induced damage, (b) back side view with minimal chipping, and (c) side wall images from microscope and SEM.

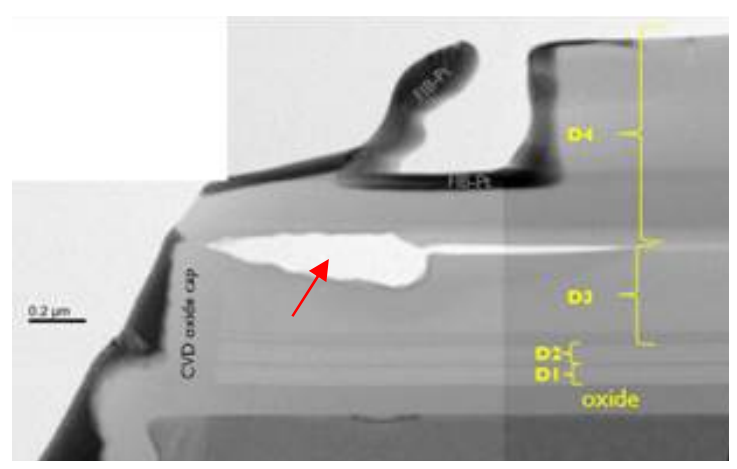

Figure 21. TEM image showing a void extending as a crack between D4 and D3 layers pointed by an arrow.

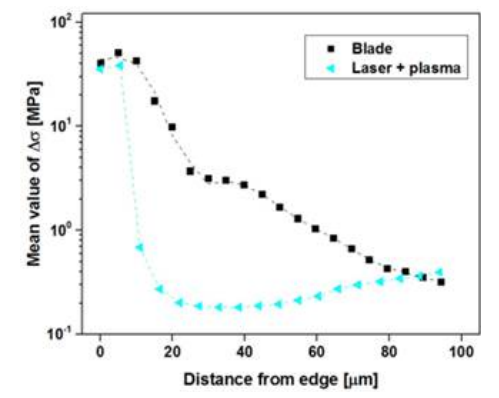

(a) Stress decay plot for blade diced and laser grooving + plasma diced sample.

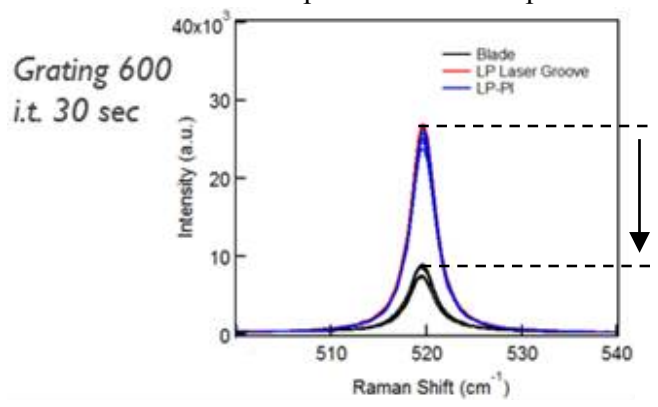

(b) Micro-Raman intensity plot for blade and two zones in combined technique: at laser grooved oart (LP Laser Groove) and plasma part (LP-PI)

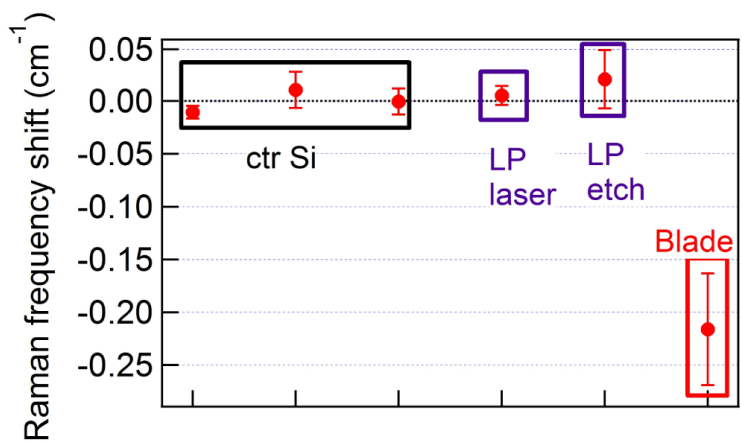

(c) Raman frequency shift $\left(\mathrm{cm}^{-1}\right)$. Showing no shift from ctr Si.

Figure 22. Stress measurements: (a) stress decay plot, (b) MicroRaman intensity plot, and (c) Raman frequency shift $\left(\mathrm{cm}^{-1}\right)$ 


\section{G. Different dicing technologies/techniques for different $3 D$ integration schemes}

The aforementioned 3D integration schemes were D2D, D2W and W2W. Different dicing techniques come into play in building these schemes: a wafer needs to be singulated in order to end up with D2D or D2W configuration; and a W2W configuration will always have to be singulated in the end to allow packaging. The suitability of a dicing technique is influenced by different factors such as the bonding method used, for instance dielectric bonding or hybrid bonding or polymer bonding. Also, it is influenced by structure and material types in the dicing street and the area in close proximity with it: the presence of (ultra) low-k dielectrics, thick or thin metal dummies, the dimension of the dicing street, etc.

For the $\mathrm{D} 2 \mathrm{D} / \mathrm{D} 2 \mathrm{~W}$ configuration, dicing takes place before bonding. Taking into consideration a polymer bonding and hybrid bonding, it is for this reason very important that particles introduced by dicing are kept minimal. This is required to achieve a good bonding interface and connection. For this purpose, laser dicing techniques or a combination with them can be suitable since this involves a coating process and removal of the coating material after dicing, which can help in having less particles after dicing. Plasma dicing is also a candidate, provided the dicing street is free of metal. Coating/development/removal of resist can be done in a metal ring frame configuration, and/or the wafer should be designed for plasma dicing. Referring to the results on plasma dicing in this study, one of the important lessons learned is the difficulty of the said technique to be integrated in a standard dicing process flow. Hence, the use of the DBG method. It was found that one of the limitations of this process is getting rid of the $\mathrm{Si}$ particles that are inserted in between the dies, given that the evaluation material has only a $10 \mu \mathrm{m}$ dicing width. It is important to note a post dicing clean is required regardless of the dicing techniques if particle requirement is strict.

Taking into account the presence of ultra low-k dielectrics, stealth dicing showed very good results. However, this technique is expected to be not plausible for the $\mathrm{W} 2 \mathrm{~W}$ configuration, particularly when dealing with multiple stacks. This is because this configuration will have alternating layers of $\mathrm{Si}$ wafer, dielectrics, metal dummies etc. Furthermore, the Si wafers can have different crystal orientations and can be as thin as $5 \mu \mathrm{m}$.

For the $\mathrm{W} 2 \mathrm{~W}$ scheme, combined dicing technique are expected not to be that useful because the dicing process will take a lot of time due to required switching in between dicing techniques. Laser full cut and blade technology can be used but with certain limitations for low-k dielectrics. Plasma dicing can also be used provided the wafers are designed for it or if the dicing flow integration (coating, development, and removal of photoresist) is addressed.

\section{SUMMARY}

From visual inspection results (optical microscope, SEM, and TEM), stealth dicing and plasma dicing clearly showed no dicing induced damage on ultra low-k or low-k layers.

Based on stress related measurements, plasma dicing and the combination of laser grooving + plasma dicing induced low stress. Diced processed using these two techniques in effect have high die strength value.

Plasma dicing was found to be very difficult to integrate into the in-house standard dicing process flow or standard module flow because coating/development/removal of resist could not be done on a wafer mounted on dicing tape and on metal ring frame configuration. The DBG method used to solve this problem is not a preferred route given a $10 \mu \mathrm{m}$ gap in between dies.

Based on the results and learnings of the different dicing techniques and processes, compatibility and challenges of dicing techniques were laid out in connection with the different 3D integration schemes.

\section{ACKNOWLEDGMENT}

The authors would like to thank all our dicing demo partners, metrology partners, and the whole 3D imec team.

\section{REFERENCES}

[1] M. Fuegl, et. al., "Assessment of dicing induced damage and residual stress on the mechanical and electrical behavior of chip", IEEE ECTC, San Diego, 2015, pp. 214-219.

[2] A. Hooper, et. al., "Review of wafer dicing techniques for via-middle process 3DI/TSV ultrathin silicon device wafers", IEEE ECTC, San Diego, 2015, pp. 1436-1446.

[3] Martin Herms, et.al., "Materials characterization and device analysis for evaluation of semiconductor processes by highly-sophisticated photoelestic stress measurement technique", Phys. Status Solidi C, vol 12, issue 8, Aug 2015, pp. 1085-1089.

[4] M. Fuegl, et. al, "Analytical stress characterization after different chip separation methods", Microelectronics Reliability, vol. 54, 2014, pp. $1735-1740$

[5] M. Kubata, et. al., "Evaluation of silicon fracture strength dependence H.D. Geiler, et. al., "Photoelastic stress evaluation and defect monitoring in 300-mm-wafer manufacturing", Material Science in Semiconductor Processing, vol. 5, 2003, pp.445-455.

[6] M. Smith et. al, "The origins of pressure-induced phase transformations during the surface texturing of silicon using femtosecond laser irradiation", Jouranl of Applied Physics, vol 112, 2012.

[7] M.Y. Tsai, and C.H. Chen, "Evaluation of test methods for silicon die strength", Elsevier Microelectronics Reliability, vol. 48, 2008, pp. 933-941

[8] Jianhua, L, et. al., "Laser dicing and subsequent die strength enhancement tehcnologies for ultra-thin wafer", IEEE ECTC, 2008, pp. 761-766.

[9] Masayoshi, K., "Advanced dicing technology for semiconductor wafer-stealth dicing", IEEE Transactions on Semiconductor Manufacturing, vol. 20, no. 3, Aug 2007, pp. 259-265.

[10] Hanxie, Z. and Dianne S., "Process challenges in low-k wafers dicing”, IEEE Electronics Manuracturing Technology Symposium, 2003, pp. 401-407. 
[11] I. de Wolf, "Micro-Raman spectroscopy to study local mechanical stress in silicon integrated circuits", Semiconductor Science
Technology, vol. 11, 1996, pp. 139-154. 